\title{
ARCHITECTURES FOR DELIVERING PROACTIVE NETWORK SERVICES IN DIVERSE BUSINESS ENVIRONMENTS
}

\author{
Don Allen \\ Ph.D. \\ Cisco Systems, Inc. \\ 14765 Pristine Drive, Colorado Springs, CO 80921, USA
}

\begin{abstract}
The introduction and adoption of cloud-based, as-a-service capabilities have had a dramatic impact on the network service management capabilities and the software architectures required to address the varied deployment requirements of customers. Historically customers were constrained, by cost, regulations, or technology, on how they designed and deployed their networks. In those environments customers deployed network management systems to manage the operations of their IT capabilities composed of vendor and opensource software. These systems were typically reserved for business-critical systems and had an operating cost above and beyond those of the business systems themselves. These support systems initially provided basic monitoring and alerting and over time expanded to include high value services such as security checks, compliance checks, policy management, and health monitoring. Over the previous decade there has been a move to migrate not only business capabilities but also the associated support systems into the cloud to reduce both software and administrative costs. This migration has not been ubiquitous across all industries due to regulatory, compliance, and security concerns as well as the nature of the company's inherent business. This has driven the need for network service management companies to develop architectures that adapt to these new deployment models. This paper identifies 4 canonical service deployment architectures under development to address the diversity of on premise, cloud, in-a-box, and hybrid business system deployment models.
\end{abstract}

\section{KEYWORDS}

Network Management, Architectures, Services, Software

\section{INTRODUCTION}

There have been several changes in the network management industry that are forcing new architectures to be considered, designed, and deployed. Network management systems are overlays on top of operating networks that are designed to ensure the availability of the network. Ensuring availability takes many forms; identifying potential vulnerabilities, misconfigurations, hardware failures, performance issues, predictions of future issues, etc. The availability and adoption of cloud-based as-a-service capabilities, the increased scrutiny on network security, company and individual data privacy, and managing overall risk have resulted in a diverse set of customer network architectures. It is not uncommon to see multiple network architectures deployed within a single, moderate size customer. Designers of network services that overlay or integrate into this application architectures have had to establish new engagement architectures when considering how to support the evolving landscape. Historically, network management systems have employed a central controller, deployed on customer premise, which utilized secure shell remote connections to a network element or a network element to controller push model, to obtain information from the network element. The central controller processed the data and would raise an alarm, send a notification, or provide some visualization of the data to an operator. As more and more network elements become more full-fledged software applications, opening remotely callable APIs, the network products are becoming more "open" and management agents deployed on those elements more capable. This presents a challenge to network management tool developers to utilize these newfound capabilities in ways that the support customer deployment constraints/requirements, interoperability, and DevOps. 
The rapid expansion of internet access and use over the last decade has placed a significant burden on companies serving the larger markets (such as Facebook, Google, Amazon, etc.). In these web-scale companies the traditional network monitoring tools that raise alarms requiring human response are too expensive and slow to address the expectations of the customers using the provided services. These web-scale companies have moved to a highly homogenous network, compute, and storage environment and have had some success in developing autonomous, self -healing systems with automated remediation of some machine detected problems. Facebook has developed Facebook Auto Remediation (FBAR) service (Komorn, 2011), LinkedIn has developed Nurse (Sherwin, 2015), Google has developed several tools based on their Site Reliability Engineering principles with the goal of maintaining an always on/almost always on set of services. These capabilities all have a common core requirement in order to be able to provide automatous network management - homogeneity across their systems. This homogeneity keeps costs low, allows the reuse of automation across multiple "products", and target specific skills and knowledge needed to operate their systems. As the benefits of these types of systems have become more widely known across the industry, non-web-scale companies have become to request/demand comparable capabilities from their technology vendors. The net-scale inhouse management architects are typically composed 3 components; 1) Monitor, 2) Analyze, and 3) Act and focus on address operational issues with the service. The monitor component looks for events, analyze judges where the issue is "real", and if so tell the act component to perform some operation (such as restarting, adding, or removing a service component.

Cisco Systems, Inc. is a \$50B company with hundreds of thousands of customers and over 60,000 partners. Cisco developers and supports networking, wireless and mobility, security, collaboration, data center, and internet of things products to customers across all business segments; financial, manufacturing, service provider, government, etc. Customers are looking to Cisco to develop network management solutions that will deliver the autonomous detection and remediation capabilities seen in the web-scale companies' implementations.

This paper will provide an overview of the capabilities that need to be deployed in a services-focused management framework across heterogeneous customer engagements and introduces four customer engagement architectures that are being used in the design of network service management software within Cisco Services to support the changing customer network deployments.

\section{SERVICE NETWORK MANAGEMENT FUNCTIONAL CAPABILITIES}

The business of providing network services to deployed environments has traditionally been focused on providing break-fix support, software upgrades, and professionals services (mostly providing expertise to customers for whatever they are willing to pay for within required margins). As networks have become more complex and expansive, new service-oriented capabilities have begun to be developed to reduce some of the growing complexity and to automate the more redundant, human intensive tasks and offload those to machines. To discuss automation in the context of architecture, it is relevant to describe the functional capabilities that must be addressed in those architectures.

A complete functional view of a network management architecture would require a considerable amount of space and is not required for this discussion. For descriptive purposes this paper will consider what constitutes the "back bone" capabilities required to overlay network management services on an operational network. This are represented in Figure 1 and described in the following section.

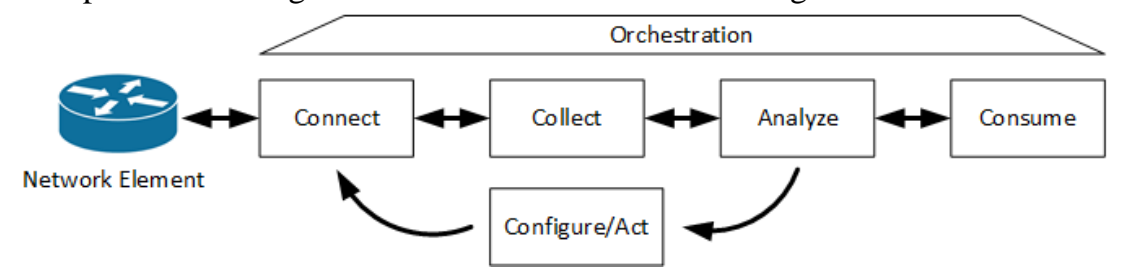

Figure 1. Backbone service management capabilities

\subsection{Network Element}

For the purposes of the discussion in this paper a network element is any network device (physical or virtual) or any application providing a service that requires the network to function. Examples of a device are a switch, router, server, firewall, etc. and networked service would be a call manager, media server, identity service, etc. 


\subsection{Connect}

To collect data from and change the configuration of a network element some type of connection to the network element must exist. In network service management a connection has historically be in the form of a remote ssh to a device or via SNMP. Over the past several years network hardware and software vendors have been introducing software agents into the network element operating systems or application programmable interfaces (APIs) to access and operate on network elements remotely. The current environments mirror a bit the systems administration environment in the late 1980's and 1990's (Nwana, 1996). Network element connectivity can be managed directly through a connection to between the network element and a cloud API or through a product or service management controller (see Figure 2). Access to and management of the connectivity can be at the network element or controller level, increasing the flexibility required in service management architectures and the complexity to software lifecycle of those architectures.

\subsection{Collect}

There are two types of data available from a network element that need to be collected to support different types of analysis; in-motion and at-rest. In-motion data is the data that represents the operation of the services provided by the network element (e.g., packet flow, response time, syslog events, etc.) and the context in which that network element is operating (e.g., CPU or memory utilization, temperature, fan speed, etc.). At-rest data refers to the configuration of a network element such as which features are turned on and how those features are configured for use. Typically, at-rest data changes are infrequent and so have different collection, change tracking, transport, storage, and analysis requirements. At-at rest data for large, multi-component devices, and network wide solutions can be substantive in size (e.g. in the hundreds of megabytes).

The data available requirements to support the application of $\mathrm{AI} / \mathrm{ML}$ and the response time required to maintain system availability are driving the need to place more analysis on the network element and to only collect the minimum data needed. Data collection requirements to support the different types of automation management must be aggregated and reconciled across the different consuming functions to minimize the network element resources used to gather and then transmit the data.

Data security, industry and government compliance, privacy issues, and transmission costs are all factors that impact what data is collected and where it is stored, the form it is stored in (anonymized or raw), and who can access it. Much of the network data needed to be collected for analysis contain potential negative business impact should a breach occur, and so customers are very protective of this data.

\subsection{Analyze}

As noted early, there are a multitude of services that can be delivered to support the continued operation and availability of network systems and these services are difficult at times to separate. In Gartner's Magic Quadrant for Network Performance Monitoring and Diagnostics (Ganguli and Corbett, 2019) the authors identify three primary focus areas of network performance monitoring and diagnostics tools; 1) digital experience monitoring, 2) networked application monitoring, and 3) network component interactions where each of their performance areas would provide analysis looking at real-time, historical, and predictive analysis. The focus areas combined with the type of analysis perform present a diverse set of requirements when it comes to collecting, aggregating, analyzing, and presenting results across a diverse set of network elements.

A discussion on network analysis would not be complete without some mention of the impact of Artificial Intelligence (AI) and Machine Learning (ML) on the future of network management. The opportunities for the application of $\mathrm{AI} / \mathrm{ML}$ are quite broad while at the same time quite challenging. Developing ML base models to support predictive analysis require large training data sets, the more complex the environment the more data is required. The larger the training data the greater the computation power required to develop the base models and test them. The cost and uniqueness of the resources required preclude many businesses from having them available to them on prem and many network management vendors are beginning to offer cloud-based analysis capabilities (e.g., Cisco DNAC).

Analysis of network element data should be performed closer to the element itself to avoid the resources needed to transmit it. Pushing the data to the cloud uses network capacity as well transmitting a lot of data which in the end has operational value since it is typically "normal". 


\subsection{Consume}

The need to make a change on a network element to remediate a detected issue requires components who have the proper access to a device and the appropriate permissions to execute the requisite actions. Historically few network element changes have been permitted by customers due to the potential impact on network or networked application availability due the inability of such changes to adequately address the complexity of the and interdependencies among network element. The use of command line interface to make configuration changes have been problematic in the past; the introduction of Netconf and Yang-based management capabilities with APIs have improved on previous states and have promise to automate a greater number of situations where automated issue resolution can be applied.

\subsection{Orchestration}

The overall control of the different network management capabilities across the network elements in a customer deployment is complex, requiring the coordination of distributed activities at the different deployment "layers". The orchestration capabilities required in a network management system start at the network element layer in monitoring and maintain the connection to a network element and end at the management of a configuration change to "like" devices across a multi-site network. There are no systems today capable of addressing the breadth of change, across the diversity of network elements, and perform that at the scale required for enterprise and service provider networks.

\subsection{Configure/Act}

The modification of a network element in response to a changing operations need (e.g., initial configuration, configuration change, on element data collection) requires the ability to make changes to the device configuration or to change the operational state of the device (e.g., start, stop, restart, etc.). Changes to a device can be made by a human or a management component and can be made through a remote command line interface (CLI) interaction or through an API (e.g., REST API, etc.).

\section{DEPLOYMENT ENVIRONMENT}

Customer networks have and continue to evolve from complete on-premise deployments where network management capabilities were bolted onto existing networks to the deployment of network elements that have been instrumented to integrate with either on premise or cloud-based management capabilities. The old deployments architectures have been supplanted by the introduction of as-a-service capabilities deployed in multi-cloud, private cloud, hybrid cloud, and hybrid (cloud and on-premise) architectures (Froehlich, 2018). The market has also seen an increase in the resources available on network elements to deliver the capabilities that previously had to be deployed on the network management controllers. Network management capabilities can now be found in the cloud, on controllers, and even on the network elements themselves. This section will describe the potential targets for deploying network service management capabilities identified in Figure 2.

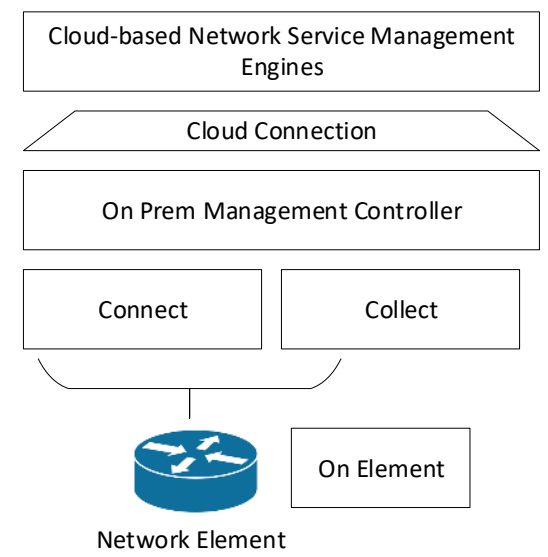

Figure 2. Deployment targets 


\subsection{On Element}

Network devices (e.g., routers, switches, wireless access points, security devices) and networked solutions (e.g., call managers, collaboration suites, calendaring solutions) are providing additional resources to enable management services to be deployed and operated. These resources are limited and therefore are typically allocated to supporting data collection, simple analysis (e.g., parsing or RegEx execution), and temporary storage. Large devices have the potential to allocate more resources to network management activities, however their allocation and use could reduce the capacity of the device. Network device operating systems and application operating systems may include their own network management capabilities that can be accessed by management controllers via APIs which both reduces the amount of software that must be developed by the network management vendor and restricts what a vendor can do with a network element.

\subsection{On-Prem Management Controller}

On-prem management controllers can take many forms but all are common in their aggregation of network elements into a single management space. There management controllers access multiple network elements to collect, analyze, share results, and configure elements. On-premise management controllers can operate independently from the cloud with tightly controlled cloud access, or in a model where some capabilities are executed on prem and others in the cloud. On-prem management controllers can communicate with each other such as a network management controller utilizing another controller to collect network element data, analyze the data, and pass the results back to that initial controller to consume. Customers have demanded more integration among on-prem controllers as well as with existing in-inhouse develop capabilities because of the cost associated with connecting network elements to the management controllers.

\subsection{Cloud-Based Service Management Engines}

Network management capabilities are moving to the cloud in the form of Software-as-a-Service (SaaS) following the model utilized by Google Apps, ServiceNow, and DropBox and also utilizing Platform-as-a-Service (PaaS) provided by Amazon Web Services, Microsoft Azure, and Google Cloud Services. Network elements can be connected to these cloud-based capabilities either directly or through On-Prem Management Controllers and the "back-bone" capabilities consolidated in the cloud or distributed across the cloud, the on-prem management controller, and on the element.

A cloud-based service management system must expose the capabilities it provides via a set of APIs and provide highly secure protection of any customer data retained on that service. Network management services are dependent on the data from network elements deployed within a customer network, as well as those in cloud SaaS and PaaS deployments.

\section{ENGAGEMENT ARCHITECTURES}

Customer network architectures are unique to a customer. Small commercial customers tend to have very similar architectures with minor variations while large enterprise and service provider networks tend to be quite varied and distinct. In addition, larger company network architectures cannot be easily characterized as a "single" deployment architecture, typically there are multiple business-solution specific architectures within the larger company network architecture, each managed by a separate organization. Network management solutions providers must develop their capabilities to accommodate the variability and complexity of these environments. The single monolithic and highly integrated software architecture must migrate to one that maintains an internal operating structure but can be loosely coupled to all the other capabilities distribute across devices, on prem controllers, cloud providers and the network management controller. When designing functional architectures to address the different deployment models thinking in terms of everything needs to connect to everything and be deployable everywhere presents a huge hurdle to designing and implementing these systems. The development of different canonical engagement architectures that represent the different customer deployment models have enabled our organization to begin to design network management software to address the deployment variability and the needs of customer across our addressable markets. 
Four engagement architectures have been used, three which could be considered truly canonical (Cloud Centric, Entrenched Keep, and In-a-Box) and the Hybrid-Hybrid that is more representative of what a medium to large company deployment might look like.

\subsection{Cloud Centric Architecture}

The cloud centric architecture addresses customers who have heavily invested in automating their network and application infrastructure. They want to use APIs to access network management capabilities in the cloud. These customers are heavily invested in their internal purpose-built systems and are willing to further invest in external professional services or self-invest in integrating with new capabilities. Some customers in this space will not allow vendor specific software in their infrastructure while at the same time they want to leverage the unique value that a vendor solution can bring to their operations.

Large and mid-size managed service partners fall into this bucket since they have historically heavily invested in systems to improve their efficiency in managing lots of customers. Customer expectation is that cloud services will comply with data sovereignty and protection governance based on the source location of the data and be able to audit it.

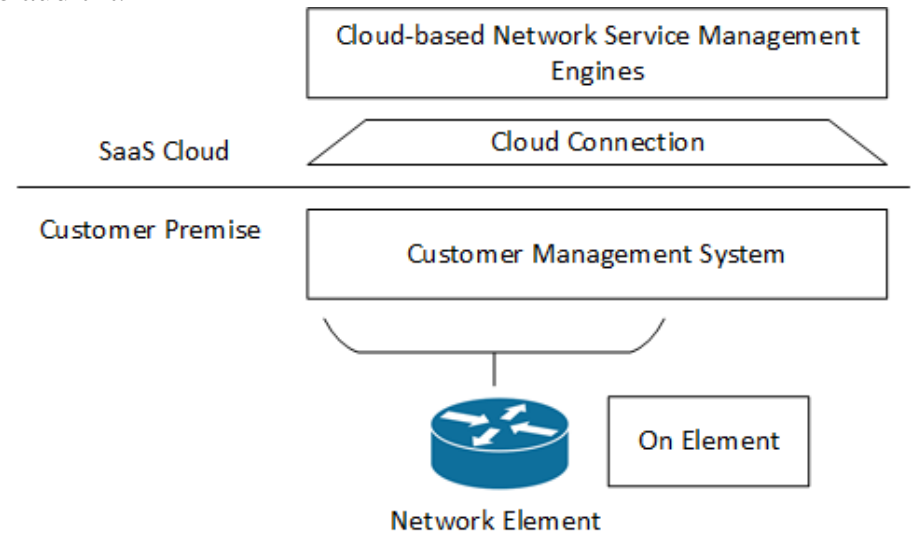

Figure 3. Cloud centric architecture

The Cloud Centric architecture (Figure 3) requires a customer to manage all network element connectivity, data collection, and configuration changes either through the customer's internal management system or connecting the device directly to the cloud-based service management engines. The cloud-based management engines expose APIs that identify the data to be collected, to receive the collected data, results, and remediations. As noted, this deployment model requires the customer to invest in the integration of their systems to the exposed management services giving the customer complete control over the software deployed in their environment and to leverage their investment in their internal systems.

\subsection{Entrenched Keep Architecture}

Customers who are very concerned about control and security because of industry/governmental oversight, potential negative impact to reputation due to a breach, or corporate fear associated with adopting new technologies. These customers do not want to send data over the internet and some do not maintain active connection to the internet. They are concerned with the potential impact of automation on the stability of their systems and want control over how a vendor capability is deployed. Customers understand that their requirements are not common and tend to over invest in custom work to address their unique requirements. Deploying vendor specific capabilities are the norm and integration across vendor solutions is expected.

Customers may use Cloud services to deploy business applications, but they maintain control of these systems, limiting their use to PaaS capabilities. Little to no information from the customer's network is sent to the cloud. On-premise controllers can use modern platform-as-a-service technologies but operate in a closed, cloud disconnected fashion. To deploy network management capabilities via an on-premise management controller the entire set of "back-bone" capabilities must be deployed and managed on the customer premise (see Figure 4). 


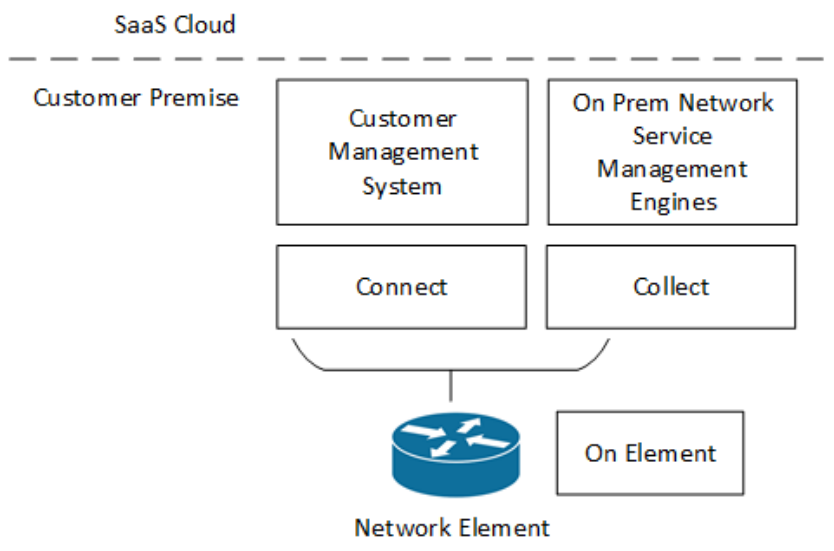

Figure 4. Entrenched keep architecture

\subsection{In-a-Box Architecture}

The In-a-Box architecture addresses the needs of most small and medium sized customers. These customers want to reap the full benefits of all the network management capabilities with minimal cost and minimal impact on their network operations. There expectations are that a network management capability can be quickly installed, value obtain with little effort, and that value can be increased as a function of the effort they invest in integrating it into their existing systems. They expect capabilities to be easily integrated into their existing products from the same vendor and with "popular" industry capabilities, either open-source of 3rd party pay-for capabilities available as cloud-based SaaS.

Customers expect incremental improvements to a capability and non-impactful upgrades and some may allow auto upgrades if there is a demonstrated track record for continued operation. These customers accept on premise or cloud-based capabilities based on where they are in their adoption of cloud-based services.

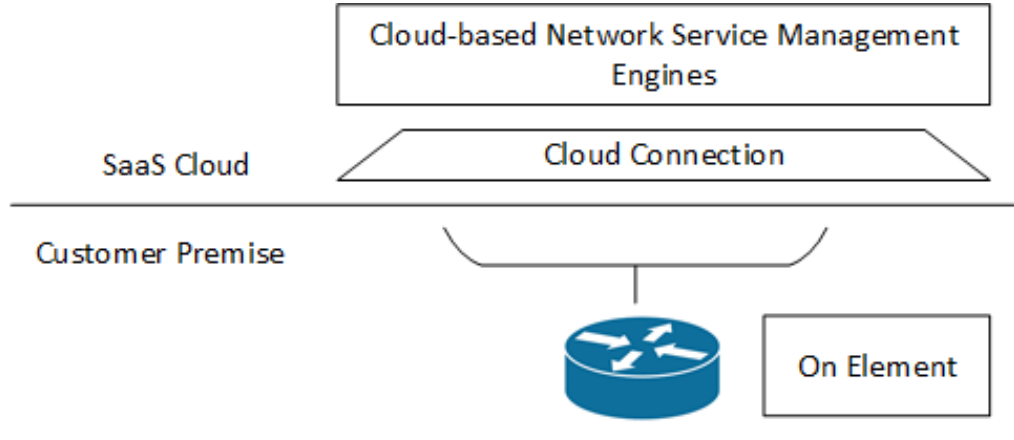

Network Element

Figure 5. In-a-box architecture

The In-a-Box architecture represented in Figure 5 deploys the network management services as a cloud-based service. Customer configure access to devices via the network management interfaces and those services can connect to the device via the internet, collect data, analyze it, present the results via a web-based user interface, and make configuration changes directly to the device. Cloud managed on prem devices have been increasing as customers look to network element vendors to take on more of the routine management of their devices. Historically in-a-box solutions were targeted at consumers or small business, however there has been an increase in the use of these architectures in components at the edge of the networks in order to reduce the operational costs required to maintain them (such as wireless access points). Automated software updates, preconfigured feature configurations, etc. are minimal requirements for in-a-box network elements. 


\subsection{Hybrid-Hybrid Architecture}

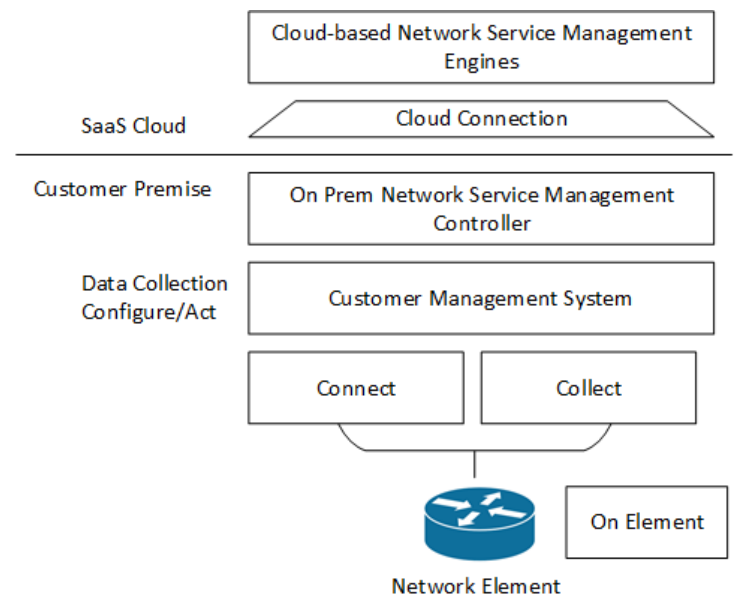

Figure 6. Hybrid-hybrid architecture

The Hybrid-Hybrid architecture conveys the need to support the deployment of different capabilities and specific features within a capability to different component. Large enterprise customers, particularly those who have a heavy cloud or internal system focus express a desire to have an on-premise network management component. This component would provide a control and communication consolidation function. This would allow to have a single point to manage access to a device and to connect those devices to the cloud-based network service management capabilities.

The hybrid-hybrid architecture in Figure 6 is an example of how backbone network service management capabilities might be distributed to the cloud, on-premise controller, and to the network element.

\section{CONCLUSION}

The introduction and adoption of cloud-based as-a-service capabilities have had a dramatic impact on the network architectures employed by customers and require changes to the architectures used by network management service providers to support them. Movement to these less expensive alternatives from historical customer network architectures is forcing network service management provides to reconsider how the design and build their software architectures to address the different customer architectures emerging. The next generation network service management capabilities must become more service/microservice based and fit with architectures that allow them to be deployed on network elements, on-premise network service management controllers, and accessed via API available in cloud-based network service management as-a-Service.

\section{REFERENCES}

Beyer, B. et al, 2016. Site Reliability Engineering: How Google Runs Production Systems. O'Reilly Media, Sebastopol, USA.

Bisordi, S. 2015. "Scaling the collection of self-service metrics [Online]". Available at https://engineering.linkedin.com/metrics/scaling-collection-self-service-metrics (Accessed June 5, 2019).

Froehlich, A. 2018. "Network Monitoring in the Cloud: 3 Options [Online]". Available at https://www.networkcomputing.com/networking/network-monitoring-cloud-3-options (Accessed 27 May 2019).

Ganguli, S and Corbett, T. 2019. Magic Quadrant for Network Performance Monitoring and Diagnostics [Online]" Available at https://www.gartner.com/doc/reprints?id=1-67RDTEE\&ct=190211\&st=sb (Accessed 15 May 2019).

Komorn, R. 2011. "Making Facebook self-healing: Automating proactive rack maintenance [Online]". Available at https://code.fb.com/production-engineering/making-facebook-self-healing-automating-proactive-rack-maintenance/ (Accessed 1 April 2019).

Nwana, H. S. 1996. "Software agents: an overview," The Knowledge Engineering Review. Cambridge University Press, 11(3), pp. 205-244.

Sherwin, B. (2015) "Introducing Nurse: Auto-Remediation at LinkedIn [Online]". Available at Introducing Nurse: Auto-Remediation at LinkedIn (Accessed 1 April 2019). 